\title{
EL USO DE VACUUM EXTRACTOR DISMINUYE LA TASA DE LESIONES MATERNAS SEVERAS ASOCIADAS AL FÓRCEPS SIN AUMENTAR LAS COMPUCACIONES FETALES SEVERAS
}

\author{
Pedro Cuevas T. ${ }^{1}$, Jorge Carvajal C. ${ }^{1}$ PhD. \\ ${ }^{1}$ Unidad de Medicina Materno-Fetal, Departamento de Obstetricia y Ginecología, Facultad de Medicina, Pontificia \\ Universidad Católica de Chile.
}

\section{RESUMEN}

Antecedentes: Existe evidencia que sugiere que el método de elección para resolver un parto operatorio es el vacuum extractor, sin embargo, esta recomendación no se ha incorporado a la práctica habitual en América Latina. Objetivos: Comparar los resultados materno-fetales de la aplicación del vacuum o fórceps. Métodos: Revisamos los 12 estudios controlados y randomizados que comparan los resultados maternofetales del uso de vacuum y fórceps, publicados hasta julio de 2006. Resultados: El vacuum como método primario es menos efectivo que fórceps en conseguir partos vaginales, sin embargo dado que permite el uso de fórceps secundario, presenta una menor tasa global de cesáreas. El vacuum extractor se asocia a una menor tasa de lesiones maternas severas, y a un aumento en la tasa de céfalo hematomas. No existen diferencias en la tasa de mortalidad perinatal ni de otras complicaciones fetales. Conclusión: El uso de vacuum se asocia a una menor morbilidad materna y a una mayor incidencia de céfalo hematoma, sin aumentar la mortalidad perinatal ni las complicaciones fetales severas.

\section{PALABRAS CLAVES: Parto vaginal asistido, fórceps, vacumm}

\section{SUMMARY}

Background: Today's evidence suggests the use of the vacuum extractor as the better method for assisted vaginal delivery; nevertheless, this recommendation has not impacted the usual practice in Latin America. Objectives: To compare the maternal-fetal outcomes associated to vacuum or forceps. Methods: We reviewed the 12 randomized controlled trials comparing the maternal-fetal outcomes of vacuum and forceps, published until July 2006. Results: The vacuum as a primary method is less effective than forceps in obtaining vaginal delivery; nevertheless, since secondary use of forceps is possible, vacuum exhibits a smaller global rate of caesarean section. The vacuum extractor is associated to a smaller rate of severe maternal injuries, and to an increased rate of cephalic hematomas. There are no differences in perinatal mortality or the rates of other fetal morbidity. Conclusion: The vacuum extractor is associated to a lower maternal morbidity and to a greater incidence of cephalic hematoma, without increasing perinatal morbidity and mortality.

KEY WORDS: Assisted vaginal delivery, forceps, vacuum extractor 


\section{INTRODUCCIÓN}

La operación cesárea se asocia a mayor morbimortalidad materna y fetal que el parto vaginal, haciendo deseable lograr, en el mayor número de los casos, un parto vaginal espontáneo (1). Existen situaciones del expulsivo que requieren de un parto vaginal asistido (2); afortunadamente el parto vaginal asistido también se asocia a menor riesgo materno que la cesárea (3).

La prevalencia del parto vaginal asistido varía entre $1,5-15 \%$, un rango amplio que probablemente refleja variaciones en las distintas escuelas de conducción del trabajo de parto (4). Enfrentados a la necesidad de un parto vaginal asistido existen 2 alternativas de uso frecuente: Vacuum Extractor o Fórceps. En América del Norte el método de elección durante años ha sido el fórceps (5), entre los argumentos planteados para esta posición destacan: la basta experiencia del equipo médico en su uso y la simpleza técnica de su utilización (6). Por su parte el vacuum es masivamente utilizado en algunas regiones de Europa (7).

Tradicionalmente se ha asociado el vacuum a una menor tasa de complicaciones maternas (8) y el fórceps a una menor tasa de lesiones fetales (9). Sin embargo, en los últimos años, probablemente gracias al desarrollo de nuevas copas de materiales suaves, ha surgido evidencia en favor de la utilización del vacuum por sobre el fórceps, reportando menor incidencia de lesiones maternas y disminución de las lesiones fetales clásicamente descritas (10).

Se hace necesario establecer con precisión las reales ventajas y desventajas de uno u otro método, permitiendo que la decisión de su uso sea basada en evidencia sólida. En este artículo revisamos la literatura para resumir la real seguridad y eficacia de ambos métodos.

\section{METODOLOGÍA}

Estrategia de búsqueda: Realizamos una búsqueda de artículos publicados en inglés o en español hasta junio de 2006 en Medline y la Base de datos Cochrane de Estudios Controlados Randomizados (CENTRAL). Se realizó una estrategia de búsqueda sensible, utilizando como palabras claves: parto vaginal asistido, fórceps y vacuum.

Selección: Seleccionamos estudios controlados randomizados, que relacionaran los eventos: parto vaginal asistido, fórceps o vacuum con los resultados de lesiones maternas y fetales según tipo de instrumento utilizado. Incluimos trabajos que eva- luaran tanto a primigestas como multíparas, con indicación de parto vaginal operatorio.

Análisis: Ambos autores evaluamos en forma independiente los trabajos que cumplían con los criterios de inclusión. Los datos crudos fueron analizados en una tabla de doble entrada, calculamos los riesgos relativos y sus respectivos intervalos de confianza. Para el análisis estadístico utilizamos un test de chi cuadrado de doble cola y consideramos para la significación estadística un valor $\mathrm{p}<0,05$.

\section{RESULTADOS}

Extracción de los datos y características de los estudios: La estrategia de búsqueda arrojó un total de 12 estudios, de los cuales 3 correspondían a publicaciones de seguimiento de artículos también incluidos en esta revisión. Se decidió incluir dichos resultados dentro de los estudios previos, quedando un total de 9 estudios para análisis. En la Tabla I se presentan, en forma resumida, las características metodológicas de cada uno.

Calidad de los estudios: Los estudios seleccionados son de calidad variable, no efectuamos análisis de heterogeneidad. Existiendo posibles sesgos dado el reducido número de pacientes incluidos y la falta de calculo de tamaño muestral en el estudio de Fall y cols (11), la inclusión de partos prematuros que pueden influir en resultado perinatal en el trabajo de Dell y cols (12), Johanson y cols (13), Williams y cols (14), Johanson y cols (15) y Bofia y cols (16) y la falta de doble ciego objetivada en todos los estudios (Tabla I).

Análisis cuantitativo: Tal como se puede ver en la Tabla II el uso de vacuum extractor, como método primario, se asocia con una mayor tasa de fracasos en lograr parto vaginal. Sin embargo, ante la falla del método se puede resolver el parto vía fórceps; de este modo, el vacuum se asocia a una menor tasa de cesáreas (Tabla II).

Al evaluar las complicaciones maternas se observa una reducción estadísticamente y clínicamente significativa en la incidencia de lesiones maternas severas (desgarros $3^{\circ}$ grado o compromiso del fórnix vaginal) y en el dolor a las 24 horas postparto (Tabla II).

Respecto de las complicaciones fetales el vacuum se asocia a un aumento estadísticamente significativo en la incidencia de céfalo hematomas, sin tener diferencias con el fórceps en la tasa de lesiones craneofaciales, necesidad de fototerapia y hemorragia retiniana (Tabla III). Al evaluar el resultado perinatal se observa una tendencia al 
Tabla I

REVISIÓN CRÍTICA DE LOS ESTUDIOS INCLUIDOS EN EL ANÁLISIS

\begin{tabular}{|c|c|c|c|c|c|c|}
\hline Estudio & $n$ & Randomización & Doble ciego & $\begin{array}{c}\text { Primigestas } \\
\%\end{array}$ & $\begin{array}{c}\text { Multíparas } \\
\%\end{array}$ & Prematuros \\
\hline Fall (11) & 36 & si & no & 100 & 0 & no \\
\hline Dell (12) & 118 & si & no & 100 & 0 & si \\
\hline Johanson (13) & 264 & si & no & 72 & 28 & si \\
\hline Williams (14) & 99 & si & no & 80 & 20 & si \\
\hline Johanson (15) & 607 & si & no & 80 & 20 & si \\
\hline Bofill (16) & 637 & si & no & 59 & 41 & si \\
\hline Pliego (17) & 140 & si & no & 52 & 48 & no \\
\hline Weerasekera (18) & 442 & si & no & $n / c$ & $\mathrm{n} / \mathrm{c}$ & no \\
\hline Vacca (19) & 304 & si & no & 90 & 10 & no \\
\hline
\end{tabular}

n/c: No consignado.

Tabla II

COMPLICACIONES MATERNAS ASOCIADAS AL USO DE VACUUM O FÓRCEPS

\begin{tabular}{lccccc}
\hline Pronóstico & $\begin{array}{c}\text { Vacuum } \\
n / \text { total } \\
(\%)\end{array}$ & $\begin{array}{c}\text { Fórceps } \\
n / \text { total } \\
(\%)\end{array}$ & $\begin{array}{c}\text { Valor } \\
p\end{array}$ & $\begin{array}{c}\text { Riesgo } \\
\text { relativo }\end{array}$ & $95 \%$ IC \\
\hline Tasa falla método & $169 / 1212(13,9)$ & $115 / 1221(9,4)$ & $<0,05$ & 1,48 & $1,18-1,85$ \\
Tasa de cesárea & $15 / 711(2,1)$ & $29 / 699(4,1)$ & $<0,05$ & 0,51 & $0,27-0,94$ \\
Lesiones severas & $119 / 1249(9,5)$ & $249 / 1263(19,7)$ & $<0,05$ & 0,48 & $0,39-0,59$ \\
Dolor severo 24 horas & $21 / 247(8,5)$ & $37 / 248(14,9)$ & $<0,05$ & 0,57 & $0,34-0,94$ \\
\hline
\end{tabular}

Resultados corresponden al resumen de los datos crudos de referencias números: 11-19.

aumento de los neonatos con APGAR $<7$ a los 5 minutos y otra tendencia a una menor mortalidad perinatal en el grupo de vacuum, ambos resultados no alcanzan significación estadística (Tabla III).

\section{DISCUSIÓN}

Presentamos la evidencia disponible para conocer con certeza las ventajas y desventajas del uso de vacuum o fórceps ante un parto vaginal asistido. Los resultados presentados muestran que en términos generales el uso de vacuum se asocia a una menor tasa de complicaciones maternas severas con un resultado perinatal en todo semejante al uso de fórceps, salvo por una mayor incidencia de céfalo hematomas.

Constatamos que el vacuum resulta ser menos efectivo que el fórceps en lograr partos vaginales. Dicha menor efectividad puede deberse a una menor fuerza de tracción de las copas suaves, a errores técnicos en la aplicación de las copas, o a una menor capacidad de mantener el vacío por las copas suaves (10). Pese a lo anterior el vacuum se asocia a un menor número de cesáreas lo que podría explicarse porque ante el fracaso de un vacuum se puede realizar un fórceps; reconocemos que dicha afirmación no está claramente explicada en los estudios presentados.

Observamos una significativa menor incidencia de complicaciones maternas inmediatas, asociado a una mejor recuperación postparto, lo cual reafirma que desde un punto vista materno el vacuum extractor constituye una mejor alternativa que el fórceps. Desde el punto de vista fetal los resultados muestran que los dos métodos son esencialmente comparables. No existen diferencias estadísticamente significativas en las tasas de complicaciones fetales salvo por una mayor incidencia de céfalo hematomas en el grupo de vacuum.

Presentados los resultados a corto plazo, estimamos que existe una duda razonable respecto del resultado a largo plazo. Actualmente existen sólo dos estudios $(20,21)$ que han reportado se- 
Tabla III

COMPLICACIONES FETALES ASOCIADAS AL USO DE VACUUM O FORCEPS

\begin{tabular}{lccccc}
\hline Pronóstico & $\begin{array}{c}\text { Vacuum } \\
n / \text { total } \\
(\%)\end{array}$ & $\begin{array}{c}\text { Fórceps } \\
n / \text { total } \\
(\%)\end{array}$ & $\begin{array}{c}\text { Valor } \\
p\end{array}$ & $\begin{array}{c}\text { Riesgo } \\
\text { relativo }\end{array}$ & $95 \%$ IC \\
\hline Apgar <7 a 1 minuto & $51 / 225(2,26)$ & $48 / 197(2,4)$ & $\mathrm{NS}$ & 0,93 & $0,66-1,31$ \\
Apgar <7 a 5 minutos & $19 / 723(2,6)$ & $13 / 710(1,8)$ & $\mathrm{NS}$ & 1,43 & $0,71-2,89$ \\
Cefalo hematoma & $116 / 1269(9,1)$ & $44 / 1279(3,4)$ & $<0,05$ & 2,66 & $1,89-3,73$ \\
Lesiones craneofaciales & $112 / 1045(10,7)$ & $122 / 1025(11,9)$ & $\mathrm{NS}$ & 0,9 & $0,71-1,15$ \\
Hemorragia retiniana & $40 / 187(21,3)$ & $36 / 192(18,7)$ & $\mathrm{NS}$ & 1,14 & $0,76-1,71$ \\
Fototerapia neonatal & $35 / 833(4,2)$ & $32 / 815(3,9)$ & $\mathrm{NS}$ & 1,07 & $0,67-1,71$ \\
Mortalidad perinatal & $3 / 877(0,3)$ & $2 / 894(0,2)$ & $\mathrm{NS}$ & 1,53 & $0,26-9,13$ \\
\hline
\end{tabular}

Resultados corresponden al resumen de los datos crudos de referencias números: 11-19.

guimiento materno-fetal a 9 meses y a 5 años postparto; dichos estudios sugieren que no existen diferencias en cuanto a la incidencia de incontinencia urinaria o fecal maternas, así como tampoco en la tasa de alteraciones visuales o de desarrollo psicomotor en los neonatos. Dicha evidencia nos parece insuficiente para obtener conclusiones definitivas.

\section{CONCLUSIONES}

El uso de vacuum extractor disminuye las complicaciones maternas severas y se asocia a una mejor recuperación al compararlo con fórceps. No existen diferencias en las complicaciones perinatales entre los grupos, salvo por un aumento en la incidencia de céfalo hematoma con el uso del vacuum. Existe insuficiente información respecto al seguimiento a mediano y largo plazo de los niños sometidos a vacuum y fórceps, indicando la necesidad de nuevos estudios que evalúen dicho resultado. Es preciso reevaluar el rol que actualmente juega el vacuum en la práctica obstétrica nacional, a fin de otorgar a nuestras pacientes la mejor alternativa terapéutica ante la necesidad de un parto vaginal asistido.

\section{BIBLIOGRAFÍA}

1. Lilford RJ, Van Coeverden de Groot HA, Moore PJ, et al. The relative risks of caesarean section (intrapartum and elective) and vaginal delivery: a detailed analysis to exclude the effects of medical disorders and acute pre-existing physiological disturbances. BJOG 1990;97:883-92.

2. ACOG Committee on Practice Bulletins. Operative Vaginal Delivery. ACOG Practice Bulletin 2000;17:18.
3. Lydon-Rochelle M, Holt VL, Martin DP, et al. Association between method of delivery and rehospitalization. JAMA 2000;283:2411-6.

4. Stephenson PA. International differences in the use of obstetrical interventions. Copenhagen: WHO (EUR/ICP/MCH) 1992;112.

5. Hillier CEM, Johanson RB. Worldwide survey of assisted vaginal delivery. Int J Gynecol Obstet 1994; 47:109-14.

6. Curtin SC, Park MM. Trends in the attendant, place, and timing of births, and in the use of obstetric interventions: United States, 1989-97. Natl Vital Stat Rep 1999;47:1-12.

7. Bergsjo P, Schmidt E, Pusch D. Differences in the reported frequencies of some obstetrical interventions in Europe. Br J Obstet Gynaecol 1983;90:62832.

8. Chalmers JA, Chalmers I. The obstetric vacuum extractor is the instrument of first choice for operative vaginal delivery. Br J Obstet Gynaecol 1989;96: 505-9.

9. Fortune P, Thomas RM. Subaponeurotic haemorrhage: A rare but life-threatening neonatal complication associated with ventouse delivery. $\mathrm{Br} \mathrm{J}$ Obstet Gynaecol 1999;106:868-70.

10. Johanson R, Menon V. Soft versus rigid vacuum extractor cups for vaginal assisted delivery. The Cochrane Database of Systematic Reviews. A metaanalysis of selected randomized controlled trials comparing outcomes between rigid versus soft vacuum extraction 1999;4.

11. Fall O, Ryden G, Finnstrom K, Finnstrom O, Leijon I. Forceps or vacuum extraction? A comparison of effects on the newborn infant. Acta Obstet Scand 1986;65:75-80.

12. Dell DL, Sighler SE, Plauche WC. Soft cup vacuum extraction: a comparison of outlet delivery. Obstet Gynecol 1985;66:624-8.

13. Johanson RB, Pusey J, Livera N, Jones P. North Staffordshire/Wigan assisted delivery trial. $\mathrm{Br} \mathrm{J}$ Obstet Gynaecol 1989;96:537-544. 
14. Williams MC, Knuppel RA, O'Brien WF, et al. A randomised comparison of assisted vaginal delivery by obstetric forceps and polyethylene vacuum cup. Obstet Gynecol 1991;78:789-94.

15. Johanson RB, Rice C, Doyle M, et al. A randomised prospective study comparing the new vacuum extractor policy with forceps delivery. $\mathrm{Br} \mathrm{J}$ Obstet Gynaecol 1993;100:524-30.

16. Bofill JA, Rust OA, Schorr SJ, at al. A randomized prospective trial of the obstetric forceps versus the M-cup vacuum extractor. Am J Obstet Gynecol 1996;175(5):1325-30.

17. Pliego AR, Moncada O, Neri E, Velasco M. Evaluación de la eficacia entre parto vaginal asistido con fórceps y extractor de vacío. Ginecol Obstet Mex 2000;68:453-9.

18. Weerasekera DS, Premaratne S. A randomised prospective trial of the obstetric forceps versus vacuum extraction using defined criteria. J Obstet Gynaecol 2002;22(4):344-5.

19. Vacca A, Grant AM, Wyatt G, Chalmers I. Portsmouth operative delivery trial: a comparison of vacuum extraction and forceps delivery. $\mathrm{Br} \mathrm{J}$ Obstet Gynaecol 1983;90:1107-12.

20. Carmody F, Grant AM, Mutch L, et al. Follow-up of babies delivered in a randomized controlled comparison of vacuum extraction and forceps delivery. Acta Obstet Gynecol Scand 1986;65:763-6.

21. Johanson RB, Heycock E, Carter J, et al. Maternal and child health after assisted vaginal delivery: fiveyear follow up of a randomised controlled study comparing forceps and ventouse. $\mathrm{Br} J$ Obstet Gynaecol 1999;106(6):544-9. 